\title{
Pseudoeosinophilia with Abnormal WBC Scattergram: an Important Diagnostic Clue on Hematology Analyzer
}

\author{
Swathi C Prabhu', Anita Tahlan', Shreya Sharmal, Sarabmeet S Lehl \\ ${ }^{1}$ Department of Pathology, Government Medical College and hospital, Chandigarh, India \\ ${ }^{2}$ Department of General Medicine, Government Medical College and Hospital, Chandigarh, India
}

\begin{abstract}
Malaria is one of the most common parasitic diseases causing a significant burden on health care, especially in India. Light microscopy, the gold standard for malaria diagnosis is time-consuming especially in a setup where the caseload is high and parasite index is low. Knowledge of abnormal scattergrams by the malarial parasite is very essential to suspect malaria and screen those cases thoroughly on a peripheral smear for accurate diagnosis. A timely and accurate diagnosis is crucial to the prognosis of this disease.

Herein, we report a case of a 45-year-old male from Punjab who presented to the emergency with fever and an altered sensorium. Analyzer showed characteristic pseudo eosinophilia with greying of neutrophil eosinophil zone with double eosinophil cluster. According to flagging rules because of eosinophilia, peripheral blood film (PBF) was made and examined, which revealed Plasmodium falciparum gametocytes.
\end{abstract}

Keywords: Malaria; Sysmex; Pathology

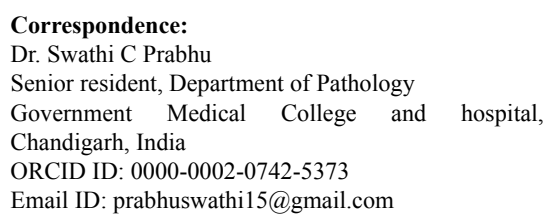

Submitted: $1^{\text {st }}$ November 2021 Accepted: $27^{\text {th }}$ December 2021

Source of Support: None

Conflict of Interest: None

Citation: Prabhu SC, Tahlan A, Sharma S, Lehl SS Pseudoeosinophilia with Abnormal WBC Scattergram: an Important Diagnostic Clue on Hematology Analyzer. NMJ 2021;4(2):502-5. DOI 10.3126/nmj.v4i2.41920

\section{INTRODUCTION}

Malaria is one of the most important parasitic diseases causing significant morbidity and mortality globally of which Plasmodium falciparum alone accounts for $>90 \%$ mortality. ${ }^{1}$ Therefore it is important to diagnose it at the earliest. $\mathrm{CBC}$ is the baseline investigation that is ordered for all cases with fever. Hence, it is essential to identify abnormal WBC scattergrams generated by malarial parasites on the analyzer. This can help us to suspect the disease and diagnose it accurately, especially in a place with a high caseload. Early and accurate diagnosis is essential for both effective management of the disease and strong malaria surveillance.

Various diagnostic methods are used for the detection of malarial parasites. Microscopic examination is the gold standard. Although a cost-effective method, it is time-consuming. Several other methods include rapid diagnostic tests (RDTs) which detect malarial antigen, fluorescence microscopy using acridine orange. However, both have limited sensitivity. Sensitive diagnostic tests include polymerase chain reaction (PCR), real-time quantitative PCR (qPCR), and reverse transcriptase PCR (RT-PCR). They are highly sensitive as they can detect $<10$ parasites $/ \mathrm{mL}$, the caveat being cost and high maintenance. ${ }^{2}$

Many studies have been conducted all over the world in the detection of the malarial parasite using automated analyzers like LH750, GEN S in the past. ${ }^{3}$ Herein, we present a case of a 45 -yearold male who presented with a fever. Investigation revealed increased eosinophils and abnormal WBC scattergram by Sysmex XN-1000 hematology analyzer. Subsequent examination of the slide showed numerous gametocyte forms of Plasmodium falciparum malarial parasite and no eosinophilia. 


\section{\# CASE REPORT}

A 45-year-old male who is a known alcoholic, intravenous drug abuser presented to the emergency with a fever. The patient also had altered bowel movements and altered sensorium for 1 day. The patient was found to be Hepatitis $\mathrm{C}$ positive. $\mathrm{CBC}$ values done on the Sysmex XN1000 analyzer on day 0 of admission were as follows: $\mathrm{Hb}: 5.7 \mathrm{gm} / \mathrm{dL}$, TLC of $9.06 \times 10^{3} / \mu \mathrm{L}$ with differential of neutrophil $41.1 \%$, lymphocytes $8.4 \%$, monocytes $3.5 \%$, eosinophils $46.9 \%$ and basophils $0.1 \%$. The platelet count was $103 \times 10^{3} / \mu \mathrm{L}$. However, differential count after slide review was as follows neutrophils $82 \%$, lymphocytes $5 \%$, monocytes $7 \%$ and eosinophils $6 \%$. The scattergram showed greying of neutrophil eosinophil region and duplication of eosinophil cluster as shown in figure 1. A normal WBC scattergram is shown in figure 2 for comparison.

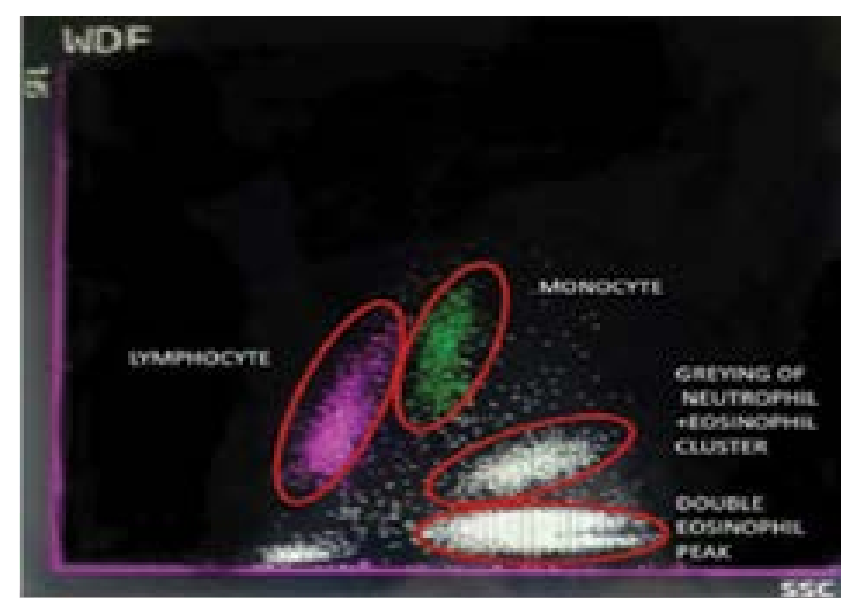

Figure 1: Scattergram generated by Sysmex XN1000 hematology analyzer from the sample of a patient with Malaria infection (Plasmodium falciparum) with double eosinophil populationpseudoeosinophilia and greying of the neutrophil-eosinophil cluster group

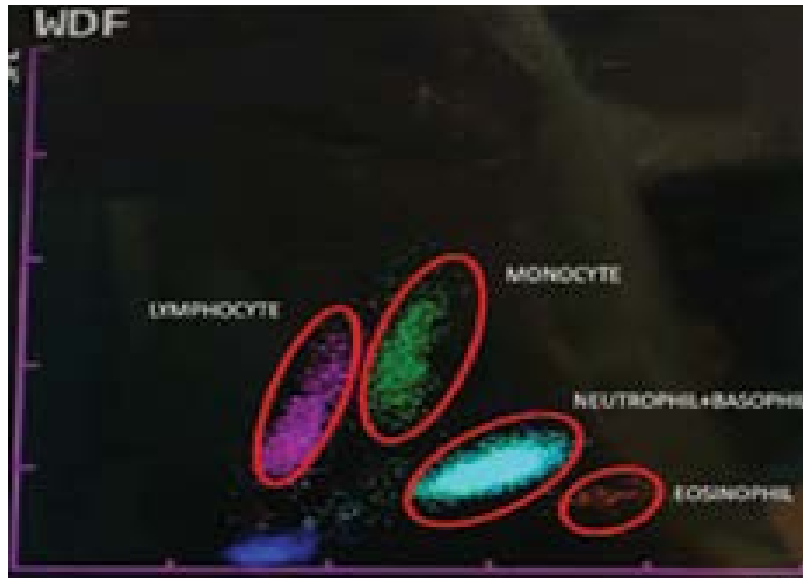

Figure 2: Normal WBC Scattergram. Note absence of doble eosinophil population

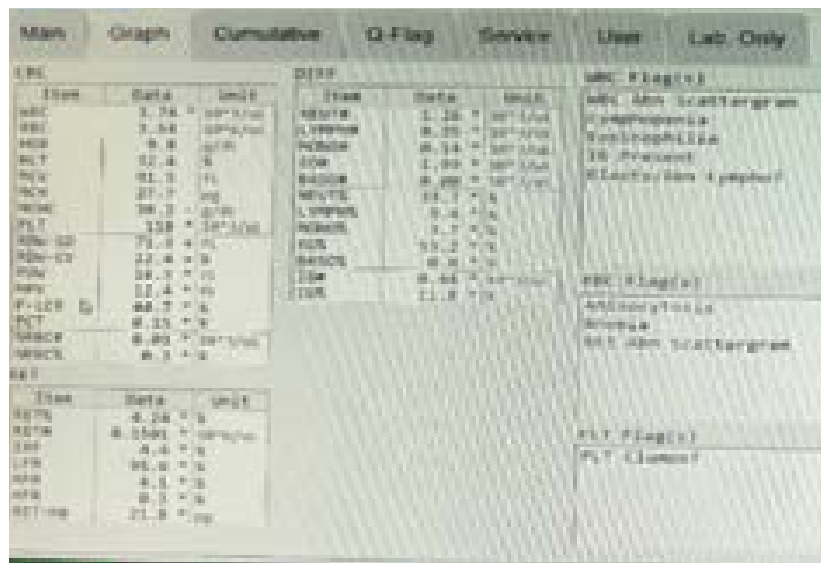

Figure 3: Pseudoeosinophilia on Sysmex XN1000

The scattergram and CBC count as shown in table 1 is of the same patient on 3 different days $(0,4$ and 11 days). The first 2 scattergrams showed erroneously high eosinophil counts and atypical eosinophils distribution in the WBCs scattergram with double eosinophil cluster and greying of the neutrophil-eosinophil region. Antimalarial treatment was started on the $4^{\text {th }}$ day with artesunate- lumefantrine till the $10^{\text {th }}$ day. Scattergram and CBC counts were completely different on the $11^{\text {th }}$ day with marked normalization of WBC scattergram and normal eosinophil count, which corresponded to a decrease in parasite density on the peripheral smear. (fig. 3 and 4) Malarial parasite density was calculated using the formula:

$\%$ parasitemia $=($ parasitized RBCs/total RBCs $) \times 100$ as given by Centre for Disease Control and Prevention. ${ }^{4}$ Parasite density on the day of admission and $4^{\text {th }}$ day was almost the same with $20000 / \mathrm{mL}$. Follow up on the $11^{\text {th }}$ day showed reduced parasite density of $100 / \mathrm{mL}$

Table 1: CBC and scattergram of the patient with Plasmodium falciparum infection on day 0 , day 4 and $11^{\text {th }}$ day of admission.

\begin{tabular}{|c|c|c|c|c|c|c|}
\hline CBC PARAMETERS & \multicolumn{2}{|c|}{$\begin{array}{l}\text { DAY } 0 \\
\text { (admission day) }\end{array}$} & \multicolumn{2}{|c|}{$\begin{array}{l}\text { DAY } 4 \\
\text { (day } 1 \text { of treatment) }\end{array}$} & \multicolumn{2}{|c|}{$\begin{array}{l}\text { DAY } 11 \\
\text { Last day of treatment }\end{array}$} \\
\hline $\mathrm{Hb}$ & $5.7 \mathrm{~g} / \mathrm{dL}$ & & $4.9 \mathrm{~g} / \mathrm{dL}$ & & $9.8 \mathrm{~g} / \mathrm{dL}$ & \\
\hline \multirow[t]{2}{*}{ TLC } & $9.06 \times 103$ & & 11.97 & & 2.66 & \\
\hline & $\begin{array}{l}\text { Analyzer } \\
\text { values }\end{array}$ & Manual & $\begin{array}{l}\text { Analyzer } \\
\text { values }\end{array}$ & Manual & $\begin{array}{l}\text { Analyzer } \\
\text { values }\end{array}$ & Manual \\
\hline Neutrophil & 41.1 & 82 & 24.5 & 81 & 33.7 & 37 \\
\hline Lymphocytes & 8.4 & 5 & 0.0 & 6 & 51.7 & 50 \\
\hline Monocytes & 3.5 & 7 & 2.6 & 8 & 11.7 & 13 \\
\hline Eosinophil & 46.9 & 6 & 72.8 & 5 & 0.4 & 0 \\
\hline Basophil & 0.1 & 0 & 0.1 & 0 & 0.0 & 0 \\
\hline Platelet & \multicolumn{2}{|c|}{$103 \times 103 / \mu \mathrm{L}$} & \multicolumn{2}{|c|}{$171 \times 103 / \mu \mathrm{L}$} & \multicolumn{2}{|c|}{$118 \times 103 / \mu \mathrm{L}$} \\
\hline
\end{tabular}




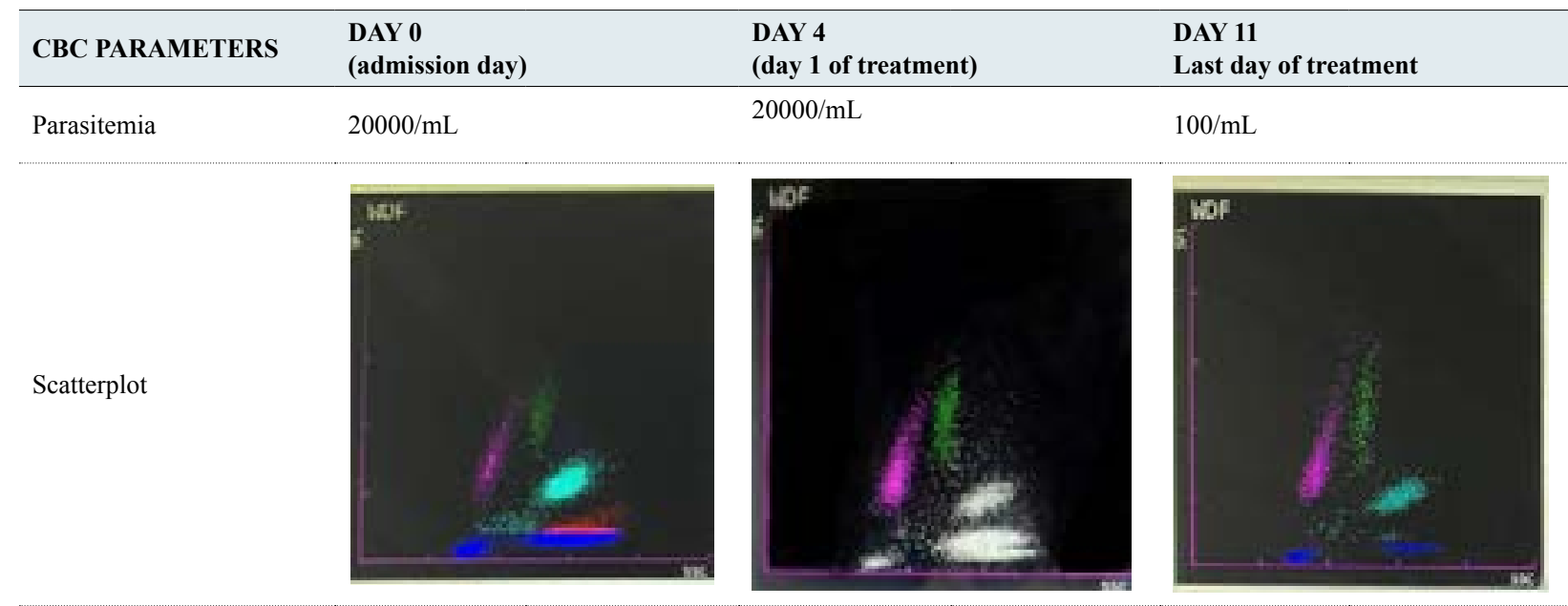

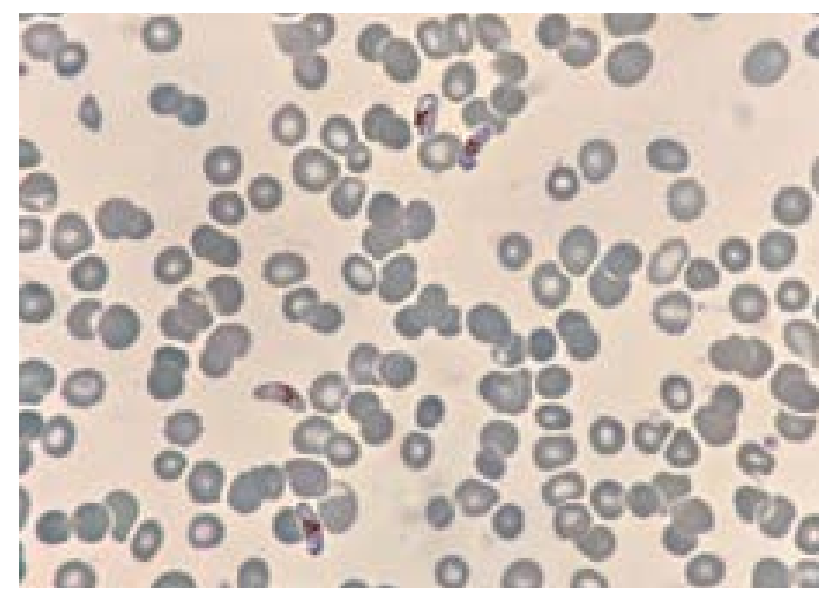

Figure 3: Photomicrograph showing gametocyte of falciparum malaria on day of admission ( Wright stain, X1000)

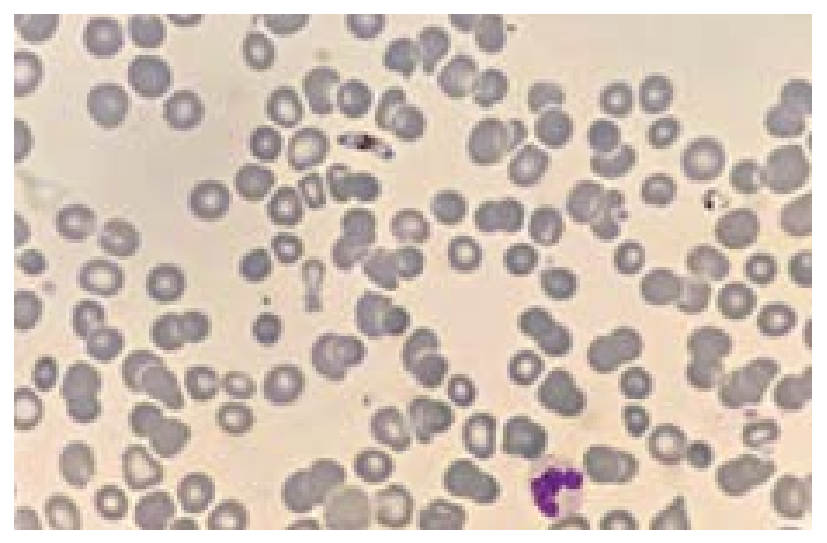

Fig 4: Photomicrograph showing a decrease in parasite density following treatment (day 11; Wright stain, X1000)

\section{DISCUSSION}

The automated cell analyzer Sysmex XN-1000 hematology analyzer is a six-part differential analyzer. It works on the principle of flow cytometry and uses semiconductor laser to give three types of optical data. Forward scatter light for the size of the cell, side scatters light denoting internal complexity of the cell, and side fluorescence light for nuclear content of the cell. ${ }^{5}$
Malaria is a highly prevalent parasitic infection that is endemic in most parts of the world and especially in India. It causes a significant burden on the health care system. ${ }^{6}$ Peripheral smear is the gold standard for the diagnosis of malaria. World health organization recommends examining 200 oil immersion fields before its declared negative on a thick smear, which takes around 20-30 minutes. This is time-consuming especially at a centre with a high turnover of cases. ${ }^{7}$ Newer techniques like RDT, Quantitative buffy coat, and Polymerase chain reaction have been introduced for malaria detection. The need for trained personnel and high cost are the most important factors preventing its widespread usage. Knowledge about scattergrams and how malarial parasites influence the changes in WBC scattergram is essential to suspect the disease. This when coupled with a thorough evaluation of the peripheral smear slide can reduce errors in the diagnosis of the disease. Early diagnosis is crucial for prognosis.

Hemazoin is the pigment that is produced by the breakdown of hemoglobin by malarial pigment.

This pigment, when engulfed by the monocytes and neutrophils, is responsible for the abnormal WBC scattergram. 8 The present case showed greying of eosinophil, neutrophil clusters, and duplication of eosinophil giving a false increase in the eosinophil.

Few studies have been done on the diagnosis of malaria based on the WBC scattergram. The most common finding was graying of both eosinophil and neutrophil groups and two eosinophil populations in around 60 percent of the cases. The rest of the least common scatterplot abnormalities were overlapping of neutrophil and eosinophil groups, two neutrophil populations, graying of both lymphocyte and monocyte groups, graying of all leucocyte groups, and two lymphocyte populations. The spurious eosinophil count was observed in $4 \%$ of the cases. ${ }^{9}$

In 2006, a Japanese study by Huh et al studied the importance of pseudoeosinophilia and WBC abnormal scattergram. They studied 144 cases of malaria and found pseudoeosinophilia in $38.9 \%$ (56 cases). The most striking abnormal WBC scattergram that was obtained was a plot extending from neutrophil to eosinophil area. ${ }^{10}$ A study by Yoho et al also found eosinophilia in 39\% of cases with $15.70 \%$ showing abnormal WBC scattergram in the form of abnormal eosinophil and neutrophil population. ${ }^{11}$ Malaria is known to cause thrombocytopenia and anemia. WBC count can also be reduced in a few cases. ${ }^{12}$ When abnormal WBC 
scattergram was used to detect the presence of malaria, sensitivity, and specificity of $83.78 \%$ and $94.82 \%$ respectively. However, in scattergram when coupled with cytopenias the sensitivity increased to $98.6 \% .^{5}$

\section{CONCLUSIONS}

All eosinophilia should be recommended for analysis of the scattergram and PBF examination. Timely treatment of malaria may help save a person's life. Therefore, this treatable cause should be searched thoroughly in a patient especially those with a history of fever. Early diagnosis may help to reduce mortality and morbidity in these patients. It may also help to contain the spread of the disease to other patients and the community. In the presence of pseudo eosinophilia and abnormal scattergram, malaria can be suspected and manual blood smear analysis should be performed for final diagnosis.

\section{Acknowledgments}

We would sincerely thank the technical personnel involved in our hematology lab for all their technical support.

\section{REFERENCES}

1. Zekar L, Sharman T. Plasmodium Falciparum Malaria. In: StatPearls [Internet]. Treasure Island (FL): StatPearls Publishing; 2021 [cited 2021 Jun 20]. Available from: Website

2. Kobayashi T, Gamboa D, Ndiaye D, Cui L, Sutton PL, Vinetz JM. Malaria Diagnosis Across the International Centers of Excellence for Malaria Research: Platforms, Performance, and Standardization. Am J Trop Med Hyg. 2015 Sep;93(3 Suppl):99-109. Crossref

3. Mendelow BV, Lyons C, Nhlangothi P, Tana M, Munster M, Wypkema E, et al. Automated malaria detection by depolarization of laser light. Br J Haematol. 1999 Mar;104(3):499-503. Crossref

4. CDC - DPDx - Diagnostic Procedures - Blood Specimens [Internet]. 2019 [cited 2021 Jun 3]. Available from: Website

5. Mohapatra S, Samantaray JC, Arulselvi S, Panda J, Munot K, Saxena R. Automated detection of malaria with haematology analyzer Sysmex XE-2100. Indian J Med Sci. 2011;65(1):26-31. Crossref

6. Levine RA, Wardlaw SC, Patton CL. Detection of haematoparasites using quantitative buffy coat analysis tubes. Parasitol Today Pers Ed. 1989;5(4):132-4. Crossref

7. Hänscheid T. Current strategies to avoid misdiagnosis of malaria. Clin Microbiol Infect Off Publ Eur Soc Clin Microbiol Infect Dis. 2003;9(6):497-504. Crossref
8. Jain M, Gupta S, Jain J, Grover RK. Usefulness of automated cell counter in detection of malaria in a cancer set up-Our experience. Indian J Pathol Microbiol. 2012 Oct 1;55(4):467-73. Crossref

9. Sharma S, Sethi N, Pujani M, Kushwaha S, Sehgal S. Abnormal WBC scattergram: a clue to the diagnosis of malaria. Hematol Amst Neth. 2013;18(2):101-5. Crossref

10. Huh HJ, Oh GY, Huh JW, Chae SL. Malaria detection with the Sysmex XE-2100 hematology analyzer using pseudoeosinophilia and abnormal WBC scattergram. Ann Hematol. 2008;87(9):755-9. $\underline{\text { Crossref }}$

11. Yoo JH, Song J, Lee KA, Sun YK, Kim YA, Park TS, Choi JR. Automated detection of malaria-associated pseudoeosinophilia and abnormal WBC scattergram by the Sysmex XE-2100 hematology analyzer: a clinical study with 1,801 patients and real-time quantitative PCR analysis in vivax malaria-endemic area. Am J Trop Med Hyg. 2010;82(3):412-4. Crossref

12. Tangpukdee N, Yew H-S, Krudsood S, Punyapradit N, Somwong W, Looareesuwan S, et al. Dynamic changes in white blood cell counts in uncomplicated Plasmodium falciparum and P. vivax malaria. Parasitol Int. 2008;57(4):490-4. Crossref 